\title{
Aseptic Loosening of Total Hip Arthroplasty
}

\author{
Infection Always Should be Ruled Out
}

\author{
Javad Parvizi MD, FRCS, Dong-Hun Suh MD, \\ S. Mehdi Jafari MD, Adam Mullan BS, \\ James J. Purtill MD
}

Received: 15 February 2010/Accepted: 10 February 2011/Published online: 2 March 2011

(C) The Association of Bone and Joint Surgeons $\mathbb{R} 2011$

\begin{abstract}
Background It is believed that some cases of aseptic failure of THA may be attributable to occult infections. However, it is unclear whether preoperative erythrocyte sedimentation rate (ESR) and C-reactive protein (CRP) are more likely elevated in these patients than those without overt infection.

Questions/purposes We asked whether some patients with aseptic THA failures have abnormal serologic indicators of periprosthetic joint infection (PJI) at the time of revision, namely ESR and/or CRP.

Methods Three hundred twenty-three revision THAs for aseptic loosening from 2004 to 2007 were retrospectively evaluated. We categorized all cases into two groups: (1) those with overt PJI $(n=14)$ plus patients who had a positive intraoperative culture during the index revision $(\mathrm{n}=13)$ and $(2)$ those who did not require rerevision $(\mathrm{n}=$ $276)$ or required surgery for noninfected causes $(n=20)$. Mean and frequency of abnormal ESR and CRP were

One or more of the authors (JP) is the consultant surgeon for Stryker Orthopaedics but he did not receive any financial support related to this article.

Each author certifies that his or her institution approved the human protocol for this investigation and that all investigations were conducted in conformity with ethical principles of research.

This work was performed at the Rothman Institute, Thomas Jefferson University, Philadelphia, PA, USA.
\end{abstract}

J. Parvizi ( $₫)$, S. M. Jafari, A. Mullan, J. J. Purtill

Department of Orthopaedic Surgery, Rothman Institute

at Thomas Jefferson University, 925 Chestnut Street,

Philadelphia, PA 19107, USA

e-mail: parvj@aol.com

D.-H. Suh

Orthopaedic Department, Korea University, Ansan Hospital,

Ansan, South Korea compared between the two groups. The minimum followup was 11 months (average, 35 months; range, 11-54 months). Results The mean and frequency of abnormal CRP in first group $(\mathrm{n}=27)$ at $2.1 \mathrm{mg} / \mathrm{dL}$ and $48 \%$ respectively, were greater than those of the uninfected $(\mathrm{n}=296)$ at $1.2 \mathrm{mg} / \mathrm{dL}$ and $27 \%$, respectively. However, there were no differences between two groups regarding mean or frequency of abnormal ESR.

Conclusion Some patients with presumed aseptic loosening may have abnormal serologic indicators of PJI that either have escaped diagnosis or were not adequately investigated. All patients undergoing revision THA should have ESR and CRP measured preoperatively and those with abnormal CRP should have additional evaluations to rule out or confirm PJI.

Level of Evidence Level III, retrospective comparative study. See the Guidelines for Authors for a complete description of levels of evidence.

\section{Introduction}

Deep PJI after total joint arthroplasty is a major complication with substantial financial and emotional burden on the healthcare system and the patient $[5,6,22]$. Despite the availability of numerous diagnostic criteria, identification of PJI is still challenging in some cases, because there is no gold standard for diagnosis [9-11, 15, 23]. Reliance on isolation of the infecting organism(s) by conventional culture cannot be used as the sole criteria for diagnosis of PJI because culture-negative PJI cases may be encountered with incidences estimated at 7\% (60 of 897 episodes of PJI) [4] to $18 \%$ (14 of 79 infected cases) [28]. Similarly, falsepositive cultures may be seen in $13 \%$ of cases [2]. However, it is critical that the diagnosis of PJI be made and 
distinction between infection and aseptic loosening is made because surgical treatment for these patients is dramatically different.

One of the main reasons for inability to isolate the infecting organism relates to the presence of a biofilm [7, 20] and/or internalization of the organism by osteoblasts $[18,20]$. Biofilm is a complex structure comprising microorganisms enveloped in macromolecules of glycocalyx and other protective films [7, 16]. Recent discoveries indicate that microorganisms in the biofilm communicate using sophisticated molecular signaling mechanisms that allow them to determine the optimal time to become planktonic [13, 26, 28]. Because isolation of a pathogen from the fluid or tissue around an infected joint mostly relies on detecting planktonic organisms, cases of culturenegative infection occur. The inability to culture organisms encased in a biofilm has led some investigators to seek biofilm disruption techniques such as sonication [21, 29]. Sonication of retrieved implants suggests bacteria were present in 77\% of so-called aseptic cases [12]. Therefore, it is plausible that some cases of subclinical infection masquerade as aseptic failure [9, 10, 20], and inability to isolate the infecting organisms may lead to implementation of surgical interventions that may be inappropriate. Some patients may have had indicators of PJI that had not been considered important.

We asked whether some patients with aseptic THA failures have abnormal serologic indicators of PJI at the time of revision, namely ESR and/or CRP.

\section{Patients and Methods}

We searched the joint arthroplasty database at our institution to identify patients who had revision THA between 2004 and 2007. An operation was considered a revision THA when any part of the hip prosthesis, including acetabular component (shell or cage), acetabular liner, femoral stem, and/or femoral head, was exchanged. Of 610 revision THAs performed during the previously mentioned period, 323 were performed on 314 patients who initially had been diagnosed with aseptic loosening. These 314 patients constituted the cohort for this study. The cohort consisted of $133(42 \%)$ men and $181(58 \%)$ women. The mean age of the patients was 67 years (range, 30-92 years) and mean body mass index was $28 \mathrm{~kg} / \mathrm{m}^{2}$ (range, $16-48 \mathrm{~kg} / \mathrm{m}^{2}$ ). The minimum followup was 11 months (average, 35 months; range, 11-54 months). The medical records of all patients were reviewed to extract detailed pertinent information. No patient was recalled specifically for this study. We had prior approval of our Institutional Review Board.

At our institution since 2004, the algorithmic approach [23] has been used routinely as part of the workup plan for patients with failed prosthetic joints. This protocol includes performing preoperative evaluations of ESR and CRP and intraoperative cultures for all patients undergoing revision joint arthroplasty. Before 2004, there was no standard protocol and any preoperative or intraoperative evaluations were obtained at the surgeon's discretion. ESR greater than $30 \mathrm{~mm} /$ hour and CRP greater than $1 \mathrm{mg} / \mathrm{dL}$ were considered abnormal [25].

Before revision surgery, all patients routinely were given intravenous first-generation cephalosporin (or vancomycin for those with a history of allergy to penicillin or cephalosporins) before skin incision and also for the first 48 hours after surgery or until the intraoperative culture results became available. All revisions were performed with the patient in the supine position, through a direct lateral approach. Extended trochanteric osteotomy was used in 59 (18\%) hips. For 169 of 323 cases (52\%) that intraoperative cultures were obtained, the following method was used. Joint fluid was first obtained (whenever present) using a large-bore needle $(14 \mathrm{G})$ connected to a syringe and before opening the capsule/pseudocapsule. Tissue cultures from the capsule region behind the acetabulum (whenever revised), intramedullary canal (when femur was revised), and/or tissues that appeared to be infected were obtained and transferred to a sterile tube using clean instruments. A minimum of three fluid and/or tissue samples were obtained for every case. Care was exercised to minimize contact of the culture samples with the drape, gloves, or used instruments during the surgery to minimize contamination. The culture samples were transferred to the microbiology laboratory as soon as the final culture sample was obtained.

Patients generally were mobilized on the first postoperative day under the supervision of a physical therapist. Weightbearing status of the patient was determined by the operating surgeon and based on the nature of the reconstruction and other factors. The regimen for thromboprophylaxis consisted of administration of warfarin on the day of surgery, which then was continued postoperatively for 6 weeks aiming for an international normalized ratio between 1.8 and 2.0. Patients were discharged from the hospital when cleared by the physical therapist and occupational therapist. The mean length of the hospital stay was 4 days (range, 2-34 days). On discharge, 118 patients went home and 181 went to a rehabilitation facility.

Patients routinely were followed clinically and radiographically at 6 weeks, 6 months, 2 years, and then every 2 years after the procedure. We did not routinely obtain ESR and CRP determinations. AP and lateral radiographs were assessed by the senior attending physician for signs of failure (loosening, infection, wear, etc). The time from the index revision until the last clinical visit or when the failure occurred was considered the followup period. 
We considered rerevision or reoperation for any reason as a failure. The diagnosis of PJI (subsequent to index revision) was made if at least one of the following criteria was present: (1) positive intraoperative cultures on solid medium; (2) neutrophil count greater than 1760 cells $/ \mu \mathrm{L}$ and polymorphonuclear count greater than $65 \%$ in the joint aspirate [23]; or (3) presence of a sinus or an abscess.

Two hundred eighty-nine patients (89\% of the cohort) did not undergo reoperation or rerevision during the followup period. The remaining 34 patients $(11 \%)$ needed reoperations either for PJI $(n=14,41 \%)$ or aseptic loosening $(n=20,59 \%)$. Intraoperative cultures were obtained in 169 of 323 patients (Fig. 1). To answer the study's question thereafter, we categorized the patients into two groups. Group 1 consisted of the patients who required reoperation for PJI $(n=14)$ plus patients who had a positive intraoperative culture during the index revision $(\mathrm{n}=$ 13). Group 2 consisted of those who did not require additional surgery $(\mathrm{n}=276)$ or who required surgery for noninfectious reasons $(n=20)$. We presumed if the patient did not have repeat surgery with confirmed infection within the minimum 11 months followup, they had no infection.

The data regarding the values of ESR and CRP for these patients that were measured before the index revision surgery were collected, and then the mean values of these two parameters were calculated. Thereafter, the mean values of the two aforementioned groups were compared using Student's t-test. Considering the abnormal values of either ESR or CRP (whichever was abnormal), we divided the patients of each group into either normal or abnormal category. Then, we compared the frequency of abnormal serology values between Groups 1 and 2 using the chi square test. SPSS ${ }^{\circledR}$ for Windows ${ }^{\circledR} 15.0$ (SPSS Inc, Chicago, IL, USA) was used for statistical analysis.

\section{Results}

Among 323 patients who had revision THAs for aseptic reasons, 147 had abnormal serology (CRP and/or ESR) or had positive intraoperative cultures. Patients in Group 1 had a similar mean ESR ( $38 \mathrm{~mm} /$ hour) to patients in Group $2(30 \mathrm{~mm} / \mathrm{hour})$. The mean CRP value of the patients in Group 1 was higher ( $\mathrm{p}=0.01)$ at $2.1 \mathrm{mg} / \mathrm{dL}$ than that of Group 2 at $1.2 \mathrm{mg} / \mathrm{dL}$. The frequency of abnormal CRP also was higher $(\mathrm{p}<0.03)$ among patients in Group 1 at $48 \%$ compared with $27 \%$ in Group 2 . There was no difference $(p=0.18)$ in the frequency of abnormal ESR between patients in Group 1 (55\%) and Group 2 (40\%).

\section{Discussion}

Because surgery for hip arthroplasties that failed owing to infection or aseptic loosening is dramatically different, definite diagnosis of PJI is critically important. Mechanisms such as the presence of a biofilm and/or internalization of an organism by osteoblasts [3, 20, 21, 28] cause the infecting organism to escape detection by conventional methods. There is no preoperative test that has consistent $100 \%$ sensitivity and specificity. Furthermore, the absence of obvious signs of infection may mislead the surgeon to think the failure is truly aseptic, and obviate the need for additional evaluations such as aspiration or obtaining intraoperative cultures. Our study was designed to determine whether aseptic failure leading to revision arthroplasty is aseptic or if infection was not properly ruled out in these patients. We therefore asked whether some patients with aseptic THA failures have abnormal serologic indicators of PJI at the time of revision, namely ESR and/or CRP.

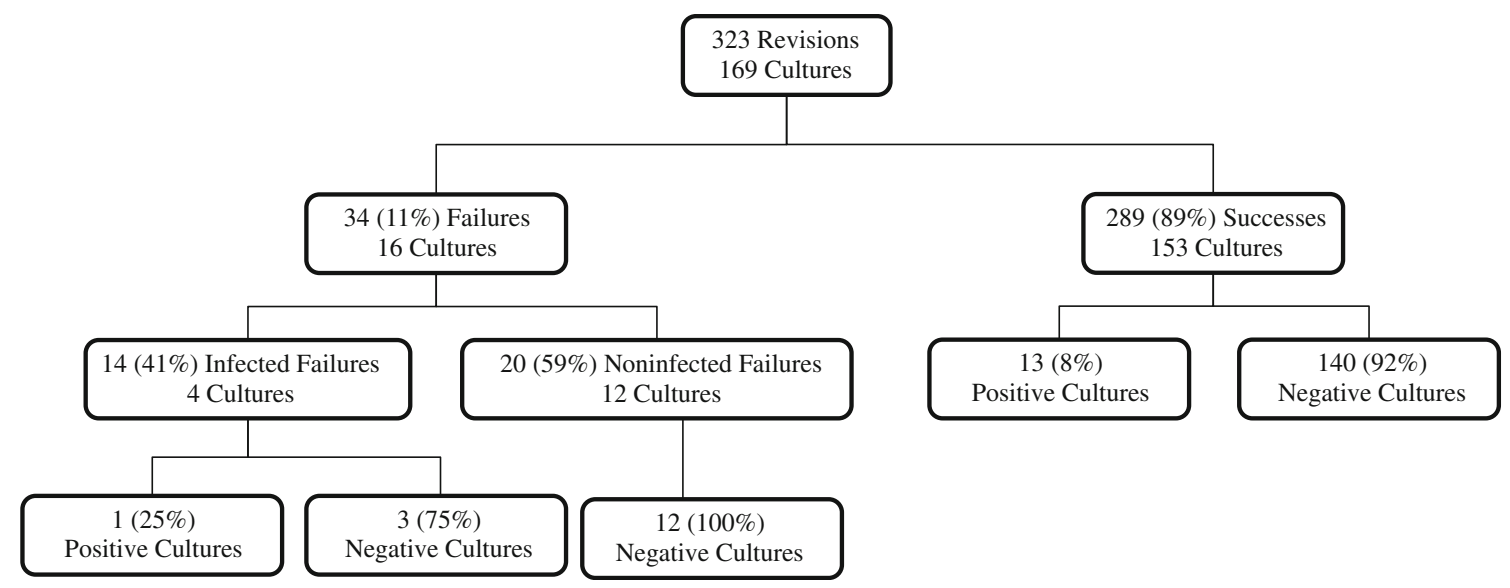

Fig. 1 The number of patients, intraoperative cultures obtained, and the positive culture results are shown in the success and failure groups. 
There are certain limitations to our study. First, not all patients undergoing revision arthroplasty at our institution were properly evaluated to rule out or confirm PJI. Therefore, it is difficult to be certain whether failure in our patients was a result of infection acquired during revision surgery of the infection was present all along and patients had been assigned erroneously to the aseptic group owing to lack of adequate evaluations for PJI. The latter point, however, is one of the main messages of the study. Patients undergoing revision for aseptic failure should receive serologic tests at a minimum and further investigations should be performed for those with abnormal serologic test results. Second, while the data were entered into a prospective database, variability in data collection may have introduced an unrecognized collection bias. Third, for the purpose of analyses, all patients with a positive intraoperative culture on solid medium were grouped as suspected PJI cases. It is possible that some of these patients may have had false-positive results and did not belong in the PJI group. We had no metrics by which to separate falsepositive from true-positive cases and therefore we used one parameter for all. Although we recognize this possibility, we believe it is remote because all cultures were obtained from deep and representative tissues during surgery and we exercised caution, such as transferring the samples directly to sterile tubes without allowing them to come into contact with gloves and drapes, to prevent contamination. Fourth, we found no difference in mean ESR values, but the groups were small, raising the possibility of a Type II error. Finally, we did not routinely obtain followup ESR or CRP values and have a minimum followup of 11 months. We therefore cannot ensure some patients considered as aseptic on followup did not have infections. However, this would not alter our conclusion that some patients underwent revision with the presumption they had no infection, and the subsequent course suggested they possibly had infections and did not have an adequate workup.

Higher mean CRP values and greater frequency of abnormal CRP values in the patients with septic failure and positive cultures suggest some of the so-called aseptic failures may be the result of subclinical infection that escaped diagnosis or were not adequately investigated. This study strengthens previous findings that showed pathogenic bacteria could be isolated from the sonicated prostheses of $1.2 \%$ of patients (three of 252 cases) with failure of their joints for what was assumed to be aseptic failure [28]. The suspicion that some of the aseptic cases are not truly aseptic is not new [12, 14]. Nelson et al. suggested that septic loosening as defined by the failure to observe clinical signs of infection and/or to isolate bacteria using standard microbiologic techniques does not completely eliminate the possibility of PJI [20]. Spangehl et al., reporting on 202 revision THAs [27], observed that ESR and CRP values do not necessarily increase concomitantly in every infected hip. As a result, increase of either ESR or CRP necessitates further investigation for infection.

Because we were concerned that some of the revisions for aseptic failures may have been the result of undetected subclinical infections, a standardized protocol was introduced in 2004 for workup of all patients awaiting revision THA [23]. The protocol mandates performing serology tests (ESR and CRP) for all patients undergoing revision arthroplasty and then implementing additional investigations such as joint aspiration and specific imaging in those with abnormal serology or high suspicion of infection. Because of high negative predictive values of ESR and CRP [27], patients with normal levels of ESR and CRP generally are not required to have additional investigations to rule out infection. It appears that based on the data from this study and a previous study [1], serology is a simple tool for screening patients suspected of PJI. However, owing to a low positive predictive value of ESR and CRP tests, we strongly recommend patients with abnormal serology undergo additional evaluations. Although more difficult and more painful than the knee, aspiration of the hip is invaluable for investigation of infection if the serology is abnormal. Analysis of the joint aspirate for absolute neutrophil count and polymorphonuclear percentage provides valuable information regarding the presence or absence of infection [19, 23, 27]. It is plausible that for some of these patients, early failure as a result of infection would have been averted if the patients had additional evaluations to rule out or confirm infection. The fact that some cases of infected failures may have escaped detection before revision also may explain the relatively high incidence of infection after revision arthroplasty observed in our study and others [8, 17, 24]. Despite the aforementioned limitations, we believe our study serves to highlight the importance of a proper workup for all patients undergoing revision THA. Based on the data from this study and from the literature, we recommend serology be performed for all patients undergoing revision THA, and subsequently patients with abnormal ESR and/or CRP undergo additional evaluation. Diagnosis of infection remains a challenge and clinical presentation rarely can distinguish between septic and aseptic failures. Thus, a valuable screening tool such as serology can avert implementation of inappropriate surgery.

\section{References}

1. Austin MS, Ghanem E, Joshi A, Lindsay A, Parvizi J. A simple, cost-effective screening protocol to rule out periprosthetic infection. J Arthroplasty. 2008;23:65-68. 
2. Barrack RL, Harris WH. The value of aspiration of the hip joint before revision total hip arthroplasty. J Bone Joint Surg Am. 1993;75:66-76.

3. Baumert N, von Eiff C, Schaaff F, Peters G, Proctor RA, Sahl HG. Physiology and antibiotic susceptibility of Staphylococcus aureus small colony variants. Microb Drug Resist. 2002;8:253-260.

4. Berbari EF, Marculescu C, Sia I, Lahr BD, Hanssen AD, Steckelberg JM, Gullerud R, Osmon DR. Culture-negative prosthetic joint infection. Clin Infect Dis. 2007;45:1113-1119.

5. Bozic KJ, Kurtz SM, Lau E, Ong K, Vail TP, Berry DJ. The epidemiology of revision total hip arthroplasty in the United States. J Bone Joint Surg Am. 2009;91:128-133.

6. Bozic KJ, Ries MD. The impact of infection after total hip arthroplasty on hospital and surgeon resource utilization. J Bone Joint Surg Am. 2005;87:1746-1751.

7. Costerton JW, Montanaro L, Arciola CR. Biofilm in implant infections: its production and regulation. Int J Artif Organs. 2005; 28:1062-1068.

8. Cui Q, Mihalko WM, Shields JS, Ries M, Saleh KJ. Antibioticimpregnated cement spacers for the treatment of infection associated with total hip or knee arthroplasty. J Bone Joint Surg Am. 2007;89:871-882.

9. Della Valle CJ, Bogner E, Desai P, Lonner JH, Adler E, Zuckerman JD, Di Cesare PE. Analysis of frozen sections of intraoperative specimens obtained at the time of reoperation after hip or knee resection arthroplasty for the treatment of infection. J Bone Joint Surg Am. 1999;81:684-689.

10. Della Valle CJ, Scher DM, Kim YH, Oxley CM, Desai P, Zuckerman JD, Di Cesare PE. The role of intraoperative Gram stain in revision total joint arthroplasty. J Arthroplasty. 1999;14: 500-504.

11. Della Valle CJ, Zuckerman JD, Di Cesare PE. Periprosthetic sepsis. Clin Orthop Relat Res. 2004;420:26-31.

12. Dobbins JJ, Seligson D, Raff MJ. Bacterial colonization of orthopedic fixation devices in the absence of clinical infection. J Infect Dis. 1988;158:203-205.

13. Dong YH, Zhang XF, An SW, Xu JL, Zhang LH. A novel twocomponent system BqsS-BqsR modulates quorum sensingdependent biofilm decay in Pseudomonas aeruginosa. Commun Integr Biol. 2008;1:88-96.

14. Dougherty SH. Pathobiology of infection in prosthetic devices. Rev Infect Dis. 1988;10:1102-1117.

15. Greidanus NV, Masri BA, Garbuz DS, Wilson SD, McAlinden MG, Xu M, Duncan CP. Use of erythrocyte sedimentation rate and C-reactive protein level to diagnose infection before revision total knee arthroplasty: a prospective evaluation. J Bone Joint Surg Am. 2007;89:1409-1416.

16. Gristina AG, Costerton JW. Bacterial adherence and the glycocalyx and their role in musculoskeletal infection. Orthop Clin North Am. 1984;15:517-535.
17. Hanssen AD, Rand JA. Evaluation and treatment of infection at the site of a total hip or knee arthroplasty. Instr Course Lect. 1999;48:111-122.

18. Jevon M, Guo C, Ma B, Mordan N, Nair SP, Harris M, Henderson B, Bentley G, Meghji S. Mechanisms of internalization of Staphylococcus aureus by cultured human osteoblasts. Infect Immun. 1999;67:2677-2681.

19. Mihalko WM, Manaswi A, Cui Q, Parvizi J, Schmalzried TP, Saleh KJ. Diagnosis and treatment of the infected primary total knee arthroplasty. Instr Course Lect. 2008;57:327-339.

20. Nelson CL, McLaren AC, McLaren SG, Johnson JW, Smeltzer MS. Is aseptic loosening truly aseptic? Clin Orthop Relat Res. 2005;437:25-30.

21. Nguyen LL, Nelson CL, Saccente M, Smeltzer MS, Wassell DL, McLaren SG. Detecting bacterial colonization of implanted orthopaedic devices by ultrasonication. Clin Orthop Relat Res. 2002;403:29-37.

22. Ong KL, Kurtz SM, Lau E, Bozic KJ, Berry DJ, Parvizi J. Prosthetic joint infection risk after total hip arthroplasty in the Medicare population. J Arthroplasty. 2009;24(6 suppl):105109.

23. Parvizi J, Ghanem E, Menashe S, Barrack RL, Bauer TW. Periprosthetic infection: what are the diagnostic challenges? J Bone Joint Surg Am. 2006;88(suppl 4):138-147.

24. Peersman G, Laskin R, Davis J, Peterson M. Infection in total knee replacement: a retrospective review of 6489 total knee replacements. Clin Orthop Relat Res. 2001;392:15-23.

25. Pulido L, Ghanem E, Joshi A, Purtill JJ, Parvizi J. Periprosthetic joint infection: the incidence, timing, and predisposing factors. Clin Orthop Relat Res. 2008;466:1710-1715.

26. Schwartz T, Walter S, Marten SM, Kirschhofer F, Nusser M, Obst U. Use of quantitative real-time RT-PCR to analyse the expression of some quorum-sensing regulated genes in Pseudomonas aeruginosa. Anal Bioanal Chem. 2007;387:513521.

27. Spangehl MJ, Masri BA, O'Connell JX, Duncan CP. Prospective analysis of preoperative and intraoperative investigations for the diagnosis of infection at the sites of two hundred and two revision total hip arthroplasties. J Bone Joint Surg Am. 1999;81: 672-683.

28. Trampuz A, Piper KE, Jacobson MJ, Hanssen AD, Unni KK, Osmon DR, Mandrekar JN, Cockerill FR, Steckelberg JM, Greenleaf JF, Patel R. Sonication of removed hip and knee prostheses for diagnosis of infection. $N$ Engl J Med. 2007;357: 654-663.

29. Tunney MM, Patrick S, Curran MD, Ramage G, Hanna D, Nixon JR, Gorman SP, Davis RI, Anderson N. Detection of prosthetic hip infection at revision arthroplasty by immunofluorescence microscopy and PCR amplification of the bacterial 16S rRNA gene. J Clin Microbiol. 1999;37:3281-3290. 